\title{
Steady-state solutions for atomic multipole moments in an arbitrarily oriented static magnetic field
}

\author{
G. Bevilacqua \\ Department of Information Engineering and Mathematical Science, University of Siena, Via Roma 56, 53100 Siena, Italy ${ }^{*}$ \\ E. Breschi and A. Weis \\ Department of Physics, University of Fribourg, Chemin du Musée 3, 1700 Fribourg, Switzerland
}

(Received 9 December 2013; published 7 March 2014)

\begin{abstract}
We derive algebraic expressions for the atomic multipole moments $m_{k, q}(F)$ describing the steady-state polarization of an atomic ensemble with angular momentum $F$ in a static magnetic field of arbitrary direction and general multipole relaxation rates $\Gamma_{q}^{(k)}$. The longitudinal moments $m_{k, 0}$ are given in terms of truncated continued fractions, while the transverse moments $m_{k, q \neq 0}$, representing the ensemble coherence, are given by products of truncated continued fractions. The special case of isotropic relaxation leads to particularly simple and elegant expressions. Our results are relevant for all domains of physics that consider the evolution of a spin system interacting with vector-type perturbations.
\end{abstract}

DOI: 10.1103/PhysRevA.89.033406

PACS number(s): $32.60 .+\mathrm{i}, 32.70 . J z, 33.40 .+\mathrm{f}, 32.80 . \mathrm{Xx}$

\section{INTRODUCTION}

The optical properties of an ensemble of independent paramagnetic atoms, such as a vapor, an atomic beam, or matrix-isolated atoms, depend on the nature, the degree, and the orientation of the ensemble's spin polarization. This fact forms the basis for a variety of atomic sensors (gyroscopes [1] and magnetometers [2]) or metrological devices such as atomic clocks [3]. In most practical applications, atomic-spin polarization is created by optical pumping [4] via angular momentum transfer from the light beam to the atoms by one or several light absorption-emission cycles.

An ensemble of independent atoms with angular momentum $F$ is fully described by the $(2 F+1)^{2}$ matrix elements $\rho_{M, M^{\prime}} \equiv\left\langle M|\rho| M^{\prime}\right\rangle$ of its density operator $\rho$, in the basis of total angular momentum eigenstates $|\mathrm{M}\rangle \equiv|\{\eta\}, F, M\rangle$, where $\eta$ is the set of quantum numbers that, with $F$ and $M$, unambiguously identify the system states. Alternatively, the density operator can be decomposed as [4-7]

$$
\rho=\sum_{k=0}^{2 F} \sum_{q=-k}^{k} m_{k, q} T_{q}^{k}
$$

in the basis of irreducible spherical tensor operators

$$
T_{q}^{k} \equiv \sum_{M, M^{\prime}}(-1)^{F-M^{\prime}}\left\langle F, M ; F,-M^{\prime} \mid k, q\right\rangle|F, M\rangle\left\langle F, M^{\prime}\right|,
$$

where the atomic multipole moments $m_{k, q}=\operatorname{Tr}\left[\left(T_{q}^{k}\right)^{\dagger} \rho\right]$ contain the same information as the matrix elements $\rho_{M, M^{\prime}}$. The hermiticity condition $\rho=\rho^{\dagger}$ translates into $m_{k, q}^{*}=$ $(-1)^{q} m_{k,-q}$.

The longitudinal multipole moments $m_{k, 0}$ can be expressed as linear combinations of the magnetic sublevel populations $\rho_{M, M}$, while the transverse moments $m_{k, q \neq 0}$ are determined by the $\Delta M=q$ coherences $\rho_{M, M-q}$.

A priori, the full system description involves as many multipole moments $m_{k, q}$ as matrix elements $\rho_{M, M^{\prime}}$. In systems

\footnotetext{
*giuseppe.bevilacqua@unisi.it
}

with a large angular momentum $F$, the number of real parameters needed to fully specify the system's polarization may thus become very large. However, this number may be substantially reduced by taking symmetries of the polarized medium into account. For instance, all transverse moments $m_{k, q \neq 0}$ vanish if the system is invariant under rotations around the quantization axis. In case the system is invariant under reflections with respect to a plane perpendicular to $\hat{z}$, all multipoles $m_{k, q}$ of odd rank $k$ will vanish.

A further reduction involves the multipolarity of the atomlight interaction. As discussed, e.g., by Happer [4], electric dipole transitions will only couple to vector moments $m_{1, q}$ and second rank tensor moments $m_{2, q}$, besides the trivial moment $m_{0,0}$ representing the total level population.

The linear absorption coefficient $\kappa$ of light coupled to an electric dipole transition in a spin-polarized medium can be written as $\kappa \propto\left|E_{0}\right|^{2} \operatorname{Tr}\left[\rho\left(\mathbf{d} \cdot \boldsymbol{\varepsilon}^{*}\right)(\mathbf{d} \cdot \boldsymbol{\varepsilon})\right]$, where the argument of the trace is given by

$$
\rho \sum_{i=1}^{3} \sum_{j=1}^{3}\left(d_{i} d_{j}\right)\left(\varepsilon_{i}^{*} \varepsilon_{j}\right) \propto \rho \sum_{k=0}^{2} \sum_{q=-k}^{k}(-1)^{q} D_{q}^{k} E_{-q}^{k},
$$

with $\mathbf{d}$ being the electric dipole operator, $\mathbf{E}=E_{0} \boldsymbol{\varepsilon}$ the optical field, and $\varepsilon$ its polarization vector. The Cartesian dyadics $d_{i} d_{j}$ and $\varepsilon_{i}^{*} \varepsilon_{j}$ are expressed in terms of the spherical operators $D_{q}^{k} \propto T_{q}^{k}$ and the tensors $E_{q}^{k}$ describing the light polarization, respectively, using standard rules. This demonstrates that the vector character of the dipole interaction implies that light can only couple to multipole moments of rank $k=0,1$ and 2 , and the nonvanishing tensor components $E_{q}^{k}$ exactly determine the multipole moments sensitive to a given light polarization.

From the above it follows that the electric dipole absorption coefficient $\kappa$ of a polarized medium can be written as

$$
\frac{\kappa}{\kappa_{\mathrm{unpol}}}=1+\sum_{k=1}^{2} \sum_{q=-k}^{k} \alpha_{q}^{(k)}\left(F, F^{\prime}\right) m_{k, q},
$$

where $\kappa_{\text {unpol }}$ is the absorption coefficient of the unpolarized medium and where we refer to the quantities $\alpha_{q}^{(k)}\left(F, F^{\prime}\right)$ as "multipole analyzing powers." These analyzing powers depend 

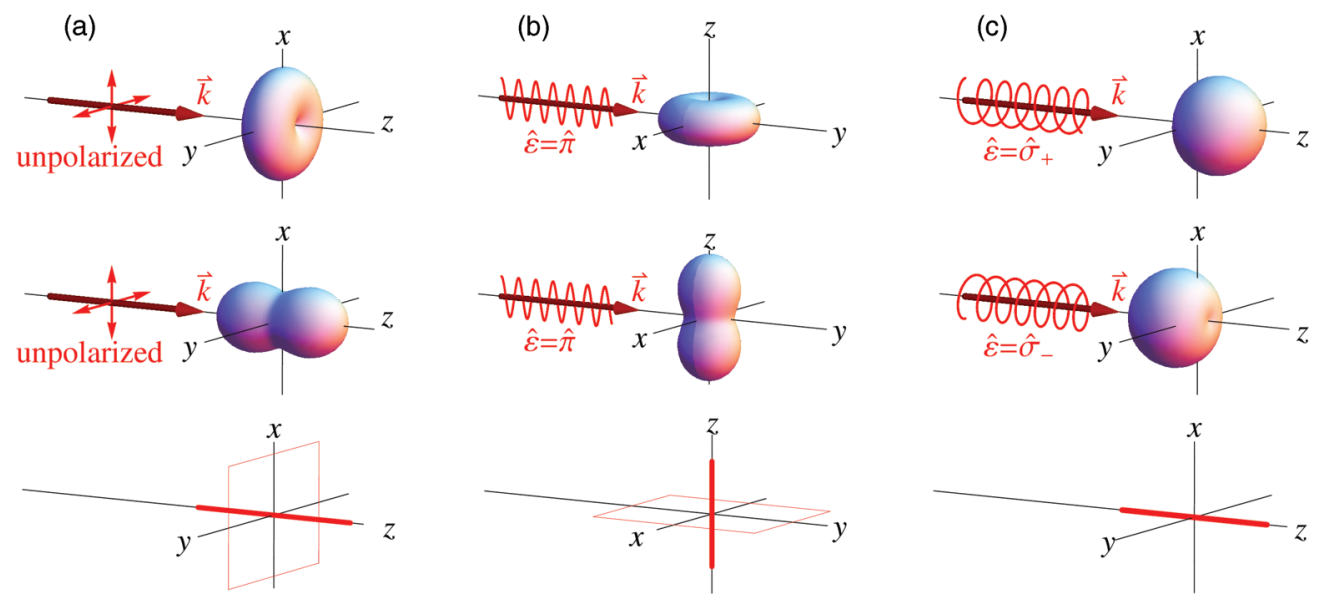

FIG. 1. (Color online) Atomic multipole moments—visualized following Refs. [8-10] - that determine the absorption of unpolarized (a), linearly polarized (b), and circularly polarized (c) light. Unpolarized light "feels" only the longitudinal alignment $m_{2,0}$ along $\vec{k}$. Linearly polarized light probes only the longitudinal alignment $m_{2,0}$ along the light's polarization vector $\hat{\varepsilon}$. Circularly polarized light senses both the longitudinal orientation $m_{1,0}$ and the longitudinal alignment $m_{2,0}$ along $\vec{k}$. For all three cases, the two upper rows represent multipole moments of opposite sign. The graphs in the lower row show the axes of rotational symmetry (chosen as quantization axis) and the planes of reflection symmetry of both the incident light and the relevant multipole moments of the medium.

on the quantum numbers $\eta$ and $F$ and $\eta^{\prime}$ and $F^{\prime}$ of the levels coupled by the dipole transition.

Let us illustrate the above for the three specific cases represented in Fig. 1.

(a) Unpolarized light is invariant under rotations around the light's propagation vector $\mathbf{k}$ and under reflections with respect to a plane perpendicular to $\mathbf{k}$ [Fig. 1(a)]. The absorption coefficient for unpolarized light thus depends only on multipole moments having the same symmetry, so that the sum in Eq. (4) reduces to a single term involving the longitudinal alignment $m_{2,0}$.

(b) Linearly polarized light has rotational and reflection symmetries with respect to the light's polarization vector $\boldsymbol{\epsilon}$ and the plane perpendicular to it, respectively. Since the symmetries are the same as in the case of unpolarized light, the absorption coefficient for linearly polarized light will also depend only on $m_{2,0}$. However, the quantization axis defining the $m_{k, q}$ is to be chosen along $\epsilon$ as shown in Fig. 1(b).

(c) Circularly polarized light has only an axis of rotational symmetry, lacking any plane of reflection symmetry. In that case the quantization axis is chosen along $\mathbf{k}$ and electric dipole transitions probe only the longitudinal moments moments $m_{1,0}$ (orientation) and $m_{2,0}$ (alignment), so that the sum in Eq. (4) reduces to two terms. Figure 1(c) represents a purely oriented medium, in which the multipoles of opposite sign are probed by light of opposite helicity.

A static magnetic field will alter both the magnitude and the orientation of the medium spin polarization. In its linear regime, the Zeeman interaction will only transform multipole moments in each subspace $k$ among themselves. In the weak light power limit, optical pumping experiments carried out by a single laser beam in the presence of a magnetic field can be well described in terms of a three-step process [11]: In a first step one considers the multipole moment $m_{k, 0}^{\text {eq }}$ prepared by optical pumping, where - as discussed above-a suitable choice of the quantization axis allows one to write the initial moment as a purely longitudinal $(q=0)$ moment. In the second step one calculates the steady-state value of $m_{k, 0}$ under the combined action of the field and the relaxation processes. The third step then consists of calculating the absorption coefficient for the same beam that has produced the initial polarization using Eq. (4), retaining only terms that reflect the symmetry of the probe light.

The present paper deals with deriving general expressions for the steady-state moments generated in the second step. We address the steady-state moments $m_{k, q}$ resulting from the precession and relaxation of an initial moment $m_{k, 0}^{\mathrm{eq}}$ in a magnetic field of arbitrary orientation. The results are general in the sense that they make no restrictions on the relaxation rates, by taking the $2 k+1$ distinct relaxation rates $\Gamma_{q}^{(k)}$ for each multipole into account.

We stress that our model and results are not limited to the atomic hyperfine structure as the notation $F$ for the angular momentum quantum number may suggest. The results presented below apply to any case in which the Zeeman Hamiltonian is linear in the magnetic field, irrespective of the total angular momentum being $F, J, L$, or $S$.

We also note that the mechanism by which the initial spin polarization is created (by optical pumping, via spin exchange by collisions with polarized atoms or other particles, by photodissociation of polarized molecules, etc.) is irrelevant for the discussion in this paper.

The paper is organized as follows. In Sec. II we give the statement of the problem; the general solution is presented in Sec. III. In Sec. IV the alignment and orientation moments are discussed in detail. In Sec. V we show the solution in the case of isotropic relaxation, and finally Sec. VI contains the conclusions.

\section{SPIN-POLARIZATION DYNAMICS IN A STATIC MAGNETIC FIELD}

In the calculations below we represent the atomic system in the basis $\{|F, M\rangle\}$ of total angular momentum states 
and set $\hbar=1$ throughout the text. The irreducible spherical tensor operators $T_{q}^{k}$ introduced in Eq. (2) obey the canonical commutation relations $\left(F_{ \pm} \equiv F_{x} \pm i F_{y}\right)$

$$
\begin{aligned}
{\left[F_{z}, T_{q}^{k}\right] } & =q T_{q}^{k}, \\
{\left[F_{ \pm}, T_{q}^{k}\right] } & =c_{ \pm}(k, q) T_{q \pm 1}^{k},
\end{aligned}
$$

where the coefficients $c_{ \pm}(k, q)$ are defined as

$$
c_{ \pm}(k, q) \equiv \sqrt{k(k+1)-q(q \pm 1)} .
$$

The dynamic evolution of $\rho$ due to the torque exerted by the static magnetic field $\mathbf{B}$ on the magnetization associated with the sample's spin polarization is governed by the Liouville equation

$$
\dot{\rho}=-i[H, \rho]-\hat{\Gamma}\left(\rho-\rho^{\mathrm{eq}}\right),
$$

where the superoperator $\hat{\Gamma}$ takes into account the effect of relaxation towards an equilibrium state described by $\rho^{\text {eq }}$. The Zeeman interaction Hamiltonian is given by

$$
\begin{aligned}
H & =-g_{F} \mu_{B} \mathbf{F} \cdot \mathbf{B} \\
& =-g_{F} \mu_{B}\left(\frac{1}{2} F_{+} B_{-}+\frac{1}{2} F_{-} B_{+}+F_{z} B_{0}\right),
\end{aligned}
$$

where $g_{F}$ is the Landé factor and $B_{0}=B_{z}$ and $B_{ \pm}=B_{x} \pm i B_{y}$ represent the longitudinal and transverse field components, respectively.

The components $F_{q}$ of the angular momentum operator $\mathbf{F}$ obey

$$
\begin{aligned}
F_{z}|F, M\rangle & =M|F, M\rangle, \\
F_{ \pm}|F, M\rangle & =c_{ \pm}(F, M)|F, M \pm 1\rangle,
\end{aligned}
$$

and are related to the spherical components by $F_{0}=F_{z}$ and $F_{ \pm 1}=\mp F_{ \pm} / \sqrt{2}$.

Inserting Eq. (1) into Eq. (7) and using the commutator relations (5), one easily derives the recursion relations

$$
\begin{aligned}
& \left(i \omega_{0} q-\Gamma_{q}^{(k)}\right) m_{k, q}+i \frac{\omega_{-} c_{+}(k, q-1)}{2} m_{k, q-1} \\
& \quad+i \frac{\omega_{+} c_{-}(k, q+1)}{2} m_{k, q+1}=-\Gamma_{q}^{(k)} m_{k, q}^{\mathrm{eq}}, \quad q=-k, \ldots, k,
\end{aligned}
$$

that describe the atomic multipole moments under steady-state conditions $\left(\dot{m}_{k, q} \equiv 0\right)$. In Eq. (10) we have expressed the magnetic field components in terms of the corresponding Larmor frequencies $\omega_{ \pm} \equiv \omega_{x} \pm i \omega_{y} \equiv g_{F} \mu_{B}\left(B_{x} \pm i B_{y}\right)$ and $\omega_{0}=\omega_{z}=g_{F} \mu_{B} B_{z} \equiv \omega_{\|}$. The relaxation superoperator is taken to be completely diagonal in the multipole basis and the hermiticity of $\rho$ requires that the relaxation rates satisfy

$$
\left(\Gamma_{q}^{(k)}\right)^{*}=\Gamma_{-q}^{(k)},
$$

as can readily checked from Eq. (10). For simplicity we neglect frequency shifts due to relaxation by assuming the relaxation rates $\Gamma_{q}^{(k)}$ to be real quantities, for which $\Gamma_{q}^{(k)}=\Gamma_{-q}^{(k)}$, so that the relaxation of a multipole of rank $k$ is characterized by $k+1$ independent rates.

By introducing the column vector

$$
\mathbf{m}=\left(m_{k,-k}, m_{k,-k+1}, \ldots, m_{k, 0}, \ldots, m_{k, k-1}, m_{k, k}\right)^{T},
$$

the recursion relations of Eq. (10) can be transformed into a system of linear algebraic equations

$$
\mathcal{H} \mathbf{m}-\mathcal{G} \mathbf{m}=-\mathcal{G} \mathbf{m}^{\text {eq }}
$$

where the $(2 k+1) \times(2 k+1)$ matrix elements of the tridiagonal Hamiltonian matrix $\mathcal{H}$ are given by

$$
\begin{aligned}
\mathcal{H}_{q, q^{\prime}}= & i \omega_{0} q \delta_{q, q^{\prime}}+i \frac{\omega_{-} c_{+}(k, q-1)}{2} \delta_{q, q-1} \\
& +i \frac{\omega_{+} c_{-}(k, q+1)}{2} \delta_{q, q+1},
\end{aligned}
$$

while the relaxation matrix $\mathcal{G}$ is diagonal with matrix elements

$$
\mathcal{G}_{q, q^{\prime}}=\Gamma_{q}^{(k)} \delta_{q, q^{\prime}}
$$

It is interesting to note that the equations of motion (13) couple the $2 k+1$ components of a multipole with given rank $k$, while they do not mix multipoles with different $k$ values. This property follows from the commutation relations (5) and from the fact that the Zeeman interaction Hamiltonian has a linear dependence on the angular momentum components $F_{q}$.

\section{GENERAL SOLUTIONS}

The multipole moments $m_{k, q}$ with $q=-k, \ldots, k$ are solutions of the system of linear algebraic equations (13) involving a tridiagonal coefficient matrix. It is well known that the matrix elements of the inverse of such a matrix can be expressed in terms of a finite continued fraction [12]. The solution takes on a particularly simple form by choosing, as stated above, the quantization axis such that the equilibrium values $m_{k, q}^{\mathrm{eq}}$ are purely longitudinal moments:

$$
m_{k, q}^{\mathrm{eq}}=\delta_{q, 0} m_{k, 0}^{\mathrm{eq}} .
$$

From Eq. (7), one sees that the $m_{k, q}^{\mathrm{eq}}$ represent the steady-state multipoles in the absence of the Zeeman interaction. When the multipole moments are produced by optical pumping with unpolarized or circularly polarized light [Figs. 1(a) and 1(b)], the equilibrium values will be along the light's propagation direction, which is then a natural choice for the quantization axis, while in the case of pumping with linearly polarized light, the natural choice of the quantization axis will be along the light polarization [Fig. 1(c)]. Below we present the solutions for the multipole moments $m_{k, q}$ in relative units,

$$
\mu_{k, 0} \equiv \frac{m_{k, 0}}{m_{k, 0}^{\mathrm{eq}}}
$$

i.e., normalized to the respective equilibrium value $m_{k, 0}^{\mathrm{eq}}$.

\section{A. Longitudinal moments}

Let us start with the solutions of Eq. (10) for the longitudinal components $m_{k, 0}$ which can be readily written in relative units as

$$
\mu_{k, 0}=\frac{-\Gamma_{0}^{(k)}}{D_{0}},
$$

where $D_{0}$ is a particular instance of the general propagator

$$
D_{n}=i n \omega_{\|}-\Gamma_{n}^{(k)}+\frac{\omega_{\perp}^{2}}{4}\left[\frac{c_{-}^{2}(n+1)}{D_{n+1}^{+}}+\frac{c_{-}^{2}(n)}{D_{n-1}^{-}}\right],
$$


with $\omega_{\perp} \equiv\left|\omega_{ \pm}\right|=\sqrt{\omega_{x}^{2}+\omega_{y}^{2}}$ representing the transverse (with respect to the quantization axis) magnetic field. The propagators $D_{n}^{ \pm}$can be evaluated recursively via

$$
D_{n}^{ \pm}=i n \omega_{\|}-\Gamma_{n}^{(k)}+\frac{\omega_{\perp}^{2}}{4} \frac{c_{ \pm}^{2}(n)}{D_{n+1}^{ \pm}}
$$

and obey

$$
\left(D_{n}^{+}\right)^{*}=D_{-n}^{-} \quad \text { and } \quad\left(D_{n}\right)^{*}=D_{-n},
$$

as a consequence of the property $c_{-}(k, n+1)=c_{-}(k,-n)=$ $c_{+}(k, n)$ of the coefficients introduced in Eq. (9b). After some algebra one obtains the continued fraction

$$
\mu_{k, 0}=\frac{1}{1-\frac{2}{\Gamma_{0}^{(k)}} \operatorname{Re}\left[\frac{\omega_{\perp}^{2} / 4 c_{-}^{2}(k, 1)}{i \omega_{\|}-\Gamma_{1}^{(k)}+\frac{\omega_{\perp}^{2} / 4 c_{-}^{2}(k, 2)}{2 i \omega_{\|}-\Gamma_{2}^{(k)}+\ddots}}\right]},
$$

where the last nonzero element is the fraction involving $c_{-}^{2}(k, n=k)$.

\section{B. Transverse moments}

Similar solutions can be derived for the transverse multipole components $m_{k, q \neq 0}$. For positive $q$ one has

$$
\begin{aligned}
\mu_{k, q}= & -\Gamma_{0}^{(k)}\left(\frac{i \omega_{-}}{2}\right)^{q} \frac{1}{D_{q}} \frac{c_{+}(k, q-1)}{D_{q-1}^{-}} \\
& \times \frac{c_{+}(k, q-2)}{D_{q-2}^{-}} \cdots \frac{c_{+}(k, 0)}{D_{0}^{-}}
\end{aligned}
$$

while for negative $q$ values one finds

$$
\begin{aligned}
\mu_{k,-|q|}= & -\Gamma_{0}^{(k)}\left(\frac{i \omega_{+}}{2}\right)^{|q|} \frac{1}{D_{-|q|}} \frac{c_{-}(k,-|q|+1)}{D_{-|q|+1}^{+}} \\
& \times \frac{c_{-}(k,-|q|+2)}{D_{-|q|+2}^{+}} \cdots \frac{c_{-}(k, 0)}{D_{0}^{+}} .
\end{aligned}
$$

Equations (21), (22), and (23) are the main results of this work. In the following section we analyze their explicit form for $k=1$ and $k=2$.

\section{LOW-ORDER MULTIPOLES: ORIENTATION AND ALIGNMENT}

We evaluate explicitly the general solution for the lowestorder multipole moments $k=1$ (orientation) and $k=2$ (alignment) that are the most relevant in experiments with optically pumped atoms.

\section{A. Longitudinal orientation $\left(\mu_{1,0}\right)$}

We obtain the steady-state longitudinal orientation $m_{1,0}$ from Eq. (21) by noting that $c_{-}^{2}(1,1)=2$, as

$$
\begin{aligned}
\mu_{1,0} & =\frac{1}{1-\frac{\omega_{\perp}^{2}}{\Gamma_{0}^{(1)}} \operatorname{Re}\left[\frac{1}{i \omega_{\|}-\Gamma_{1}^{(1)}}\right]} \\
& =\frac{\omega_{\|}^{2}+\left(\Gamma_{1}^{(1)}\right)^{2}}{\omega_{\|}^{2}+\left(\Gamma_{1}^{(1)}\right)^{2}+\omega_{\perp}^{2} \Gamma_{1}^{(1)} / \Gamma_{0}^{(1)}} .
\end{aligned}
$$

This result has the same form as Eq. (10) of Ref. [13], where the authors have derived $\mu_{1,0}$ to describe the ground-state Hanle effect based on atomic orientation. As discussed at length in that reference, the dependence of $\mu_{1,0}$ in Eq. (24) on $\omega_{\perp}$ (or, alternatively on $\left.\omega_{\|}\right)$is given by families of absorptive Lorentzian resonances, whose amplitudes (contrast) and widths depend on $\omega_{\|}\left(\right.$or $\left.\omega_{\perp}\right)$, respectively.

Before proceeding we introduce the dimensionless parameters

$$
\begin{aligned}
& \beta_{\|}=\omega_{\|} / \Gamma_{0}^{(1)}=\omega_{L} \cos \theta / \Gamma_{0}^{(1)}=\beta \cos \theta, \\
& \beta_{\perp}=\omega_{\perp} / \Gamma_{0}^{(1)}=\omega_{L} \sin \theta / \Gamma_{0}^{(1)}=\beta \sin \theta, \\
& \gamma_{1}=\Gamma_{ \pm 1}^{(1)} / \Gamma_{0}^{(1)},
\end{aligned}
$$

where $\omega_{L}=g_{F} \mu_{B}|\mathrm{~B}|$ is the Larmor frequency; where $\beta_{\perp}$ and $\beta_{\|}$represent the (dimensionless) transverse and longitudinal magnetic field components, respectively, with $\beta^{2}=\beta_{\|}^{2}+\beta_{\perp}^{2}$; and where $\theta$ is the angle between the magnetic field vector and the quantization axis.

We note that using the dimensionless notation introduced above one can show that the general expression (21) for $\mu_{k, 0}$ reduces to a rational fraction of the form

$$
\mu_{k, 0}=\frac{\sum_{i=0}^{k} b_{2 i} \beta^{2 i}}{\sum_{i=0}^{k} a_{2 i} \beta^{2 i}} .
$$

Remark on our notation for longitudinal and transverse relaxation rates. In the tensorial notation used above, the relaxation of the $k=1$ multipole components describing a spin-orientated medium are expressed by the rates $\Gamma_{0}^{(1)}$ for the (longitudinal) relaxation of sublevel populations and $\Gamma_{+1}^{(1)}=\Gamma_{-1}^{(1)}$ for the (transverse) relaxation of the $\Delta M=$ \pm 1 coherences. For historical reasons, the longitudinal and transverse relaxation rates of vector polarization are usually written as $\Gamma_{1}$ and $\Gamma_{2}$, respectively. In order to avoid confusion, we therefore retain the superscript $(k)$ when referring to relaxation rates in tensor notation. With this convention, one has $\Gamma_{0}^{(1)} \equiv \Gamma_{1}$ and $\Gamma_{ \pm 1}^{(1)} \equiv \Gamma_{2}$, and $\gamma_{1}=\Gamma_{2} / \Gamma_{1}$.

Expressed in dimensionless units, the longitudinal orientation reads

$$
\mu_{1,0}(\beta)=\frac{\gamma_{1}^{2}+\cos ^{2} \theta \beta^{2}}{\gamma_{1}^{2}+\left(\cos ^{2} \theta+\gamma_{1} \sin ^{2} \theta\right) \beta^{2}},
$$

which is of the general form (26) and which can be rewritten as

$$
\begin{aligned}
\mu_{1,0}(\beta)= & \frac{1}{1+\gamma_{1} \tan ^{2} \theta} \\
& \times\left[1+\gamma_{1} \tan ^{2} \theta \frac{\gamma_{1}^{2} /\left(\cos ^{2} \theta+\gamma_{1} \sin ^{2} \theta\right)}{\beta^{2}+\gamma_{1}^{2} /\left(\cos ^{2} \theta+\gamma_{1} \sin ^{2} \theta\right)}\right],
\end{aligned}
$$

a form which explicitly shows the Lorentzian shapes of the resonances as a function of the field amplitude $\beta$ and its direction $\theta$ and thus permits the easy identification of the resonance widths and amplitudes. 

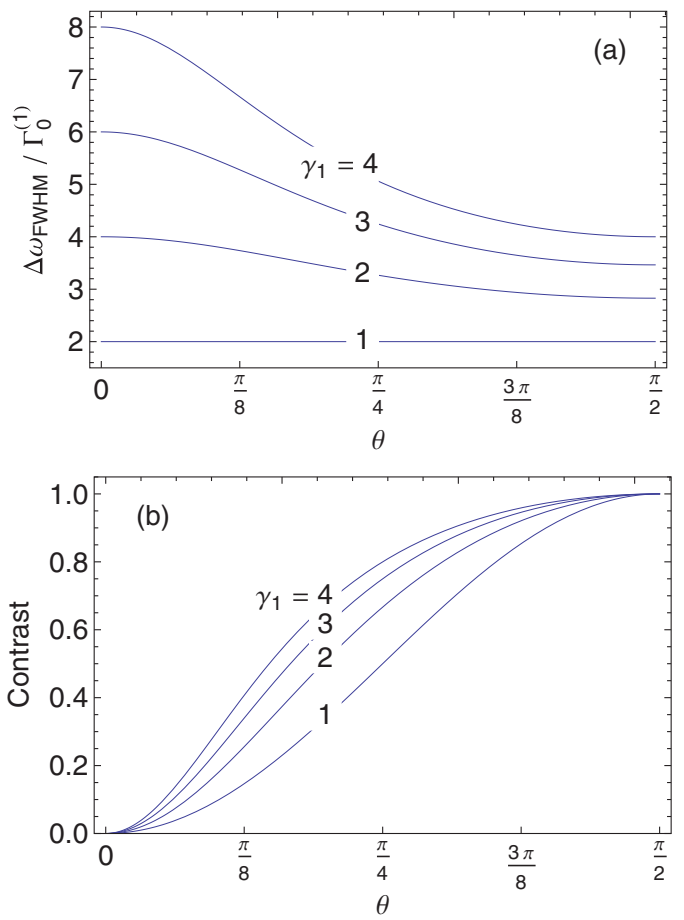

FIG. 2. (Color online) Dependence of the $m_{1,0}$ resonance width (a) and contrast (b) on the orientation $\theta$ of the scanned magnetic field with respect to the quantization axis.

In dimensionless units, the full width at half maximum $\Delta \omega_{\text {FWHM }}$ of the resonance is given by

$$
\frac{\Delta \omega_{\mathrm{FWHM}}}{\Gamma_{0}^{(1)}}=\frac{2 \gamma_{1}}{\sqrt{\cos ^{2} \theta+\gamma_{1} \sin ^{2} \theta}} .
$$

Figure 2(a) shows the dependence of the resonance width on $\theta$, the angle between the (scanned) field and the quantization axis $\hat{z}=\hat{\mathbf{k}}$. For $\gamma_{1}=1$ the width does not depend on the field orientation, while for $\gamma_{1}>1$, i.e., for $\Gamma_{1}^{(1)}>\Gamma_{0}^{(1)}$, the width is a monotonously decreasing function of $\theta$. Note that the case $\gamma_{1}<1$ is nonphysical [14]. This angular dependence of the linewidth offers an interesting approach for measuring the ratio $\gamma_{1}$ of the transverse and longitudinal relaxation rates in spin-oriented samples.

The longitudinal orientation given by Eq. (27) is bound to lie in the interval $0 \leqslant \mu_{1,0} \leqslant 1$, so that it is reasonable to define the resonance contrast as

$$
C \equiv \mu_{1,0}(\beta=0)-\mu_{1,0}(\beta \rightarrow \infty)=\frac{\tan ^{2} \theta}{\tan ^{2} \theta+\gamma_{1}^{-1}} .
$$

Figure 2(b) shows the orientation dependence of the resonance contrast that approaches asymptotically $100 \%$ for $\theta \rightarrow \pi / 2$.

\section{B. Transverse orientation $\left(\mu_{1, \pm 1}\right)$}

By applying Eqs. (22) and (23) one finds that the transverse orientation components $\mu_{1, \pm 1}$ are given by

$$
\mu_{1, \pm 1}=\mp i \Gamma_{0}^{(1)} \frac{\omega_{\mp}}{2} \frac{c_{ \pm}(0)}{D_{0}^{\mp} D_{ \pm 1}}
$$

which, after some algebra, reduces to

$$
\begin{aligned}
\mu_{1, \pm 1}= & \pm i \frac{\Gamma_{0}^{(1)} \omega_{\mp}}{\sqrt{2}} \frac{1}{\Gamma_{0}^{(1)} \pm \frac{\omega_{\perp}^{2} / 2}{i \omega_{\|} \pm \Gamma_{1}^{(1)}}} \\
& \times \frac{1}{i \omega_{\|} \mp \Gamma_{1}^{(1)}-\frac{\omega_{\perp}^{2} / 2}{\Gamma_{0}^{(1)} \pm \frac{\omega_{\perp}^{2} / 2}{i \omega_{\|} \pm \Gamma_{1}^{(1)}}}} .
\end{aligned}
$$

One can easily check by inspection that the transverse multipole moments obey $\mu_{1,1}^{*}=-\mu_{1,-1}$. Using the dimensionless variables introduced above, and assuming equal relaxation rates, one obtains the explicit dependence,

$$
\begin{aligned}
\sqrt{2} \operatorname{Re} \mu_{1, \pm 1}= & \pm \sin \theta \cos \theta \cos \varphi\left(1-\frac{1}{\beta^{2}+1}\right) \\
& \mp \sin \theta \sin \varphi \frac{\beta}{\beta^{2}+1}, \\
\sqrt{2} \operatorname{Im} \mu_{1, \pm 1}= & -\sin \theta \cos \theta \sin \varphi\left(1-\frac{1}{\beta^{2}+1}\right) \\
& -\sin \theta \cos \varphi \frac{\beta}{\beta^{2}+1},
\end{aligned}
$$

of the real and imaginary parts on the magnetic field's magnitude and orientation in the spherical coordinates $\theta$ and $\varphi$. The resonant structure of the $|\Delta M|=1$ coherences is thus given by a superposition of absorptive and dispersive Lorentzians that reduce to purely dispersive resonances for $\theta=\pi / 2$.

\section{Longitudinal alignment $\left(\mu_{2,0}\right)$}

By applying Eq. (21) one finds that the the steady-state value of the longitudinal alignment $\mu_{2,0}$ is given by

$$
\mu_{2,0}=\frac{1}{1-\frac{3 \omega_{\perp}^{2}}{\Gamma_{0}^{(2)}} \operatorname{Re}\left[\frac{1}{i \omega_{\|}-\Gamma_{1}^{(2)}+\frac{\omega_{\perp}^{2}}{2 i \omega_{\|}-\Gamma_{2}^{(2)}}}\right]} .
$$

In analogy with Eq. (25), we introduce dimensionless variables and parameters by expressing all frequencies and rates in units of the longitudinal alignment relaxation rate $\Gamma_{0}^{(2)}$ :

$$
\begin{aligned}
& \beta_{\|}=\omega_{\|} / \Gamma_{0}^{(2)}=\omega_{L} \cos \theta / \Gamma_{0}^{(2)}=\beta \cos \theta, \\
& \beta_{\perp}=\omega_{\perp} / \Gamma_{0}^{(2)}=\omega_{L} \sin \theta / \Gamma_{0}^{(2)}=\beta \sin \theta, \\
& \gamma_{1}=\Gamma_{1}^{(2)} / \Gamma_{0}^{(2)}, \\
& \gamma_{2}=\Gamma_{2}^{(2)} / \Gamma_{0}^{(2)} .
\end{aligned}
$$

After explicitly evaluating the real part of Eq. (35) the alignment can be expressed in the form

$$
\mu_{2,0}=\frac{b_{0}+b_{2} \beta^{2}+b_{4} \beta^{4}}{a_{0}+a_{2} \beta^{2}+a_{4} \beta^{4}},
$$

with coefficients

$$
\begin{aligned}
a_{0}= & b_{0}=\gamma_{1}^{2} \gamma_{2}^{2}, \\
a_{2}= & \gamma_{1} \gamma_{2}\left(3 \gamma_{2}+2\right) \\
& +\left(4 \gamma_{1}^{2}-3 \gamma_{1} \gamma_{2}^{2}+2 \gamma_{1} \gamma_{2}+\gamma_{2}^{2}\right) \cos ^{2} \theta,
\end{aligned}
$$




$$
\begin{aligned}
b_{2}= & 2 \gamma_{1} \gamma_{2}+\left(4 \gamma_{1}^{2}-2 \gamma_{1} \gamma_{2}+\gamma_{2}^{2}\right) \cos ^{2} \theta, \\
a_{4}= & 1+3 \gamma_{2}+\left(-6-6 \gamma_{2}+12 \gamma_{1}\right) \cos ^{2} \theta \\
& +\left(9-12 \gamma_{1}+3 \gamma_{2}\right) \cos ^{4} \theta, \\
b_{4}= & \left(3 \cos ^{2} \theta-1\right)^{2} .
\end{aligned}
$$

It is straightforward to verify that Eq. (37) is fully equivalent to Eq. (8) of Ref. [15], where the steady-state longitudinal alignment $m_{2,0}$ was derived to describe ground-state Hanle resonances observed with linearly polarized light.

In the Appendix we derive the explicit resonance structure of Eq. (37) involving three distinct relaxation rates. In the case of isotropic relaxation we retrieve the result

$$
\mu_{2,0}=\left(\frac{1-3 \cos ^{2} \theta}{2}\right)^{2}+\frac{3 \sin ^{2} \theta \cos ^{2} \theta}{\beta^{2}+1}+\frac{3}{16} \frac{\sin ^{4} \theta}{\beta^{2}+1 / 4},
$$

which is identical to Eq. (9) of Ref. [15].

\section{Longitudinal octupole moment $\left(\mu_{3,0}\right)$}

As above, one readily derives that the field dependence of the longitudinal octupole moment is given by

$$
\mu_{3,0}=\frac{1}{1-\frac{6 \omega_{1}^{2}}{\Gamma_{0}^{(3)}} \operatorname{Re}(L)},
$$

where

$$
L \equiv \frac{1}{i \omega_{\|}-\Gamma_{1}^{(3)}+\frac{5 \omega_{\perp}^{2} / 2}{2 i \omega_{\|}-\Gamma_{2}^{(3)}+\frac{3 \omega_{\perp}^{2} / 2}{3 i \omega_{\|}-\Gamma_{3}^{(3)}}}} .
$$

Introducing dimensionless field variables and relaxation rates as in Eq. (36), one obtains after some algebra

$$
\mu_{3,0}=\frac{b_{0}+b_{2} \beta^{2}+b_{4} \beta^{4}+b_{6} \beta^{6}}{a_{0}+a_{2} \beta^{2}+a_{4} \beta^{4}+a_{6} \beta^{6}} .
$$

The resonance structure of Eq. (42) can be examined using the method described in the Appendix for the $k=2$ case, but the algebraic expressions become too cumbersome to be of any direct practical use. However, in the case of equal relaxation rates one easily obtains

$$
\begin{aligned}
\mu_{3,0}= & \frac{1}{4} \cos ^{2} \theta\left(5 \cos ^{2} \theta-3\right)^{2}+\frac{3}{8} \frac{\sin ^{2} \theta\left(5 \cos ^{2} \theta-1\right)^{2}}{\beta^{2}+1} \\
& +\frac{15}{16} \frac{\cos ^{2} \theta \sin ^{4} \theta}{\beta^{2}+1 / 4}+\frac{5}{72} \frac{\sin ^{6} \theta}{\beta^{2}+1 / 9} .
\end{aligned}
$$

In the following section we show that the explicit results (39) and (43) derived for the low-rank multipoles $(k=1$, 2 , and 3) can be generalized for arbitrary ranks $k$ in the case of isotropic relaxation $\left(\Gamma_{q}^{(k)}\right.$ independent of $q$ ).

\section{STEADY-STATE SOLUTIONS $\mu_{k, 0}$ FOR ISOTROPIC RELAXATION}

The operator $\mathcal{H} / i$ governing the spin dynamics in the applied magnetic field is Hermitian and can thus be diagonalized by means of a unitary transformation:

$$
\mathcal{H}_{d}=U^{-1} \mathcal{H} U \text {. }
$$

The explicit form of the matrix representations of $\mathcal{H}_{d}$ and $U$ can be found easily using physical arguments. In a coordinate frame in which the quantization axis $(\hat{z})$ is along the magnetic field $\mathbf{B}$, the Hamiltonian $\mathcal{H}$ is diagonal since $\mathbf{F} \cdot \mathbf{B}$ reduces to $B F_{z}$, the matrix representation of $F_{z}$ being diagonal. Let the tilde denote quantities in the coordinate frame in which the Hamiltonian is diagonal, viz.,

$$
\left(\tilde{\mathcal{H}}_{d}\right)_{q, q^{\prime}}=\left(U^{-1} \mathcal{H} U\right)_{q, q^{\prime}}=i q \omega_{L} \delta_{q, q^{\prime}} .
$$

The unitary transformation that brings the laboratory $z$ axis to the $\hat{B}$ direction is a rotation, so that the matrix representation of $U$ which accomplishes the diagonalization is a special case of the Wigner rotation matrices [16]. When the direction of $\mathbf{B}$ is described by $\varphi$ and $\theta$ in spherical laboratory coordinates, the matrix elements of $U$ are given by

$$
U_{q, q^{\prime}}=\left\langle k, q\left|\mathrm{e}^{-i \varphi F_{z}} e^{-i \theta F_{y}}\right| k, q^{\prime}\right\rangle=e^{-i q \varphi} d_{q, q^{\prime}}^{k}(\theta),
$$

while the inverse transformation reads

$$
U_{q, q^{\prime}}^{-1}=e^{i q^{\prime} \varphi} d_{q, q^{\prime}}^{k}(-\theta) .
$$

The vector of multipole moment components $\tilde{m}_{k, q}$ in the rotated frame reads

$$
\widetilde{\mathbf{m}}=U^{-1} \mathbf{m},
$$

so that the algebraic equations of motion of the multipole components (13) become

$$
\widetilde{\mathcal{H}}_{d} \tilde{\mathbf{m}}=\widetilde{G}\left(\widetilde{\mathbf{m}}-\widetilde{\mathbf{m}}^{\mathrm{eq}}\right) .
$$

We note that the matrix $\widetilde{G}=U^{-1} G U$ is in general nondiagonal. The linear system (49) is thus by no means simpler than the original system (13). The only notable exception occurs when $G$ is proportional to the identity matrix. This holds when all relaxation rates are equal $\left(\Gamma_{q}^{(k)} \equiv \Gamma\right)$ as is the case for isotropic relaxation processes. In that case $\widetilde{G}=G$, with matrix representation $G_{q, q^{\prime}}=\Gamma \delta_{q, q^{\prime}}$, so that the solutions of Eq. (49) become

$$
\widetilde{\mathbf{m}}_{q}=\frac{\Gamma}{\Gamma-i \omega_{L} q} \widetilde{\mathbf{m}}_{q}^{\mathrm{eq}} .
$$

Using the condition (16) one finds

$$
\begin{aligned}
\left(\widetilde{\mathbf{m}}^{\mathrm{eq}}\right)_{q} & =\sum_{q^{\prime}} U_{q, q^{\prime}}^{-1}\left(\mathbf{m}^{\mathrm{eq}}\right)_{q^{\prime}}=\sum_{q^{\prime}} U_{q, q^{\prime}}^{-1} \delta_{q^{\prime}, 0}\left(\mathbf{m}^{\mathrm{eq}}\right)_{0} \\
& =U_{q, 0}^{-1}\left(\mathbf{m}^{\mathrm{eq}}\right)_{0}=U_{q, 0}^{-1} m_{k, 0}^{\mathrm{eq}},
\end{aligned}
$$

and consequently

$$
\tilde{\mathbf{m}}_{q}=\frac{\Gamma}{\Gamma-i \omega_{L} q} U_{q, 0}^{-1} m_{k, 0}^{\mathrm{eq}},
$$

so that the multipole components in the laboratory frame $(\mathbf{m}=$ $U \widetilde{\mathbf{m}})$ become

$$
m_{k, q}=m_{k, 0}^{\mathrm{eq}} \sum_{q^{\prime}} \frac{\Gamma}{\Gamma-i \omega_{L} q^{\prime}} U_{q, q^{\prime}} U_{q^{\prime}, 0}^{-1} .
$$

The longitudinal multipole moments of interest then read

$$
\mu_{k, 0}=\sum_{q} \frac{\Gamma}{\Gamma-i \omega_{L} q} d_{0, q}^{k}(\theta) d_{q, 0}^{k}(-\theta) .
$$


Using the symmetry relations $d_{q, 0}^{k}(-\theta)=d_{0, q}^{k}(\theta)$ and $d_{0, q}^{k}=$ $(-1)^{-q} d_{q, 0}^{k}$ and the reality of the Wigner matrices $d_{q, q^{\prime}}^{k}$, we finally obtain the general expression

$$
\begin{aligned}
\mu_{k, 0} & =\left|d_{0,0}^{k}(\theta)\right|^{2}+2 \sum_{q=1}^{k} \frac{\left|d_{q, 0}^{k}(\theta)\right|^{2}}{1+\left(q \omega_{L} / \Gamma\right)^{2}} \\
& =\sum_{q=-k}^{k}\left|d_{q, 0}^{k}(\theta)\right|^{2} \frac{q^{-2}}{\beta^{2}+q^{-2}},
\end{aligned}
$$

which is a sum of Lorentzian resonances, each of half width $\delta \omega_{\mathrm{HWHM}}=\Gamma / q$, that represent contributions from the $|\Delta M|=|q|=1,2, \ldots, k$ coherences and that are superimposed on a background that depends on the field orientation, but not on its magnitude. The resonance amplitudes can alternatively be expressed in terms of the associated Legendre polynomials $P_{k, 0}$ or the $q=0$ components of the spherical harmonics $Y_{k, q}$ and the spherical unit tensors $C_{k, q}$ :

$$
\begin{aligned}
\left|d_{q, 0}^{k}(\theta)\right|^{2} & =\frac{(k-q) !}{(k+q) !}\left|P_{k, 0}(\cos \theta)\right|^{2} \\
& =\frac{4 \pi}{2 k+1}\left|Y_{k, 0}(\theta, 0)\right|^{2} \\
& =\left|C_{k, 0}(\theta, 0)\right|^{2} .
\end{aligned}
$$

By direct inspection one can very that Eqs. (39) and (43) are particular cases of the general expression (55).

\section{DISCUSSION AND CONCLUSIONS}

We have presented general algebraic steady-state solutions for the atomic multipole moments $\mu_{k, q}(F)$ describing a spin-polarized ensemble of independent atoms with angular momentum $F$ interacting with a static magnetic field of arbitrary direction while relaxing. General results are given in terms of continued fractions that are then expanded into explicit expressions for the moments $\mu_{1,0}, \mu_{1, \pm 1}, \mu_{2,0}$, and $\mu_{3,0}$, the first of which are relevant for spectroscopy experiments involving electric dipole transitions.

For the particular case of isotropic relaxation, the longitudinal moments are represented by a simple sum of absorptive Lorentzian resonances with linewidths decreasing linearly with $q$. Our results may be applied to all situations in which an ensemble of independent polarized particles is subject to a vector-type interaction in the presence of relaxation.

\section{APPENDIX: MAGNETIC FIELD DEPENDENCE OF LONGITUDINAL ALIGNMENT: MOST GENERAL CASE}

\section{Arbitrary $\Gamma_{0}^{(2)}, \Gamma_{1}^{(2)}$, and $\Gamma_{2}^{(2)}$}

In this appendix we analyze explicitly the resonance structure of the field dependence of the alignment given by Eq. (37) of the main text, viz.,

$$
\mu_{2,0}=\frac{b_{0}+b_{2} \beta^{2}+b_{4} \beta^{4}}{a_{0}+a_{2} \beta^{2}+a_{4} \beta^{4}}
$$

where the coefficients $a_{i}$ and $b_{i}$ are given by Eqs. (38).
After some algebra, Eq. (A1) can be rewritten as

$$
\mu_{2,0}=\frac{b_{4}}{a_{4}}+\frac{a_{4} b_{2}-b_{4} a_{2}}{a_{4}^{2}} \frac{\beta^{2}+\frac{a_{4} b_{0}-b_{4} a_{0}}{a_{4} b_{2}-b_{4} a_{2}}}{\beta^{4}+a_{2} / a_{4} \beta^{2}+a_{0} / a_{4}},
$$

which is of the form

$$
\mu_{2,0}=P_{0}+P_{1} f(\beta) \quad \text { with } \quad f(\beta)=\frac{\beta^{2}+\xi_{2}}{\beta^{4}+\xi_{1} \beta^{2}+\xi_{0}},
$$

where $P_{0} \propto b_{4}$ is a magnetic-field-independent background which can be zeroed at the "magic" angle $\theta=\cos ^{-1}(1 / \sqrt{3})$ and where $f(\beta)$ contains the (resonant) magnetic field dependence. The latter can be decomposed according to

$$
f(\beta) \equiv \sum_{i=1}^{4} \frac{R_{i}}{\beta-z_{i}}
$$

with poles at $\beta=z_{i}$ and corresponding residues $R_{i}$. The $z_{i}$ can be expressed as

$$
\begin{aligned}
& z_{1,2}= \pm \frac{i}{\sqrt{2}} \sqrt{\xi_{1}-\sqrt{\Delta}} \equiv \pm i y_{1}, \\
& z_{3,4}= \pm \frac{i}{\sqrt{2}} \sqrt{\xi_{1}+\sqrt{\Delta}} \equiv \pm i y_{2} .
\end{aligned}
$$

By numerical inspection one finds that $\Delta=\xi_{1}^{2}-4 \xi_{0}$ and $\xi_{1} \pm$ $\sqrt{\Delta}$ are positive quantities for all relevant values of $\gamma_{1}, \gamma_{2}$, and $\theta$, so that the $z_{i}$ are purely imaginary numbers. The residues are found to be given by

$$
\begin{aligned}
& R_{1}=R_{2}^{*}=-\frac{i}{2 y_{1}} \frac{y_{1}^{2}-\xi_{2}}{y_{1}^{2}-y_{2}^{2}}, \\
& R_{3}=R_{4}^{*}=+\frac{i}{2 y_{2}} \frac{y_{2}^{2}-\xi_{2}}{y_{1}^{2}-y_{2}^{2}},
\end{aligned}
$$

so that the resonance function becomes

$$
f(\beta)=-2 \operatorname{Im}\left(R_{1}\right) \frac{y_{1}}{\beta^{2}+y_{1}^{2}}-2 \operatorname{Im}\left(R_{3}\right) \frac{y_{2}}{\beta^{2}+y_{2}^{2}} .
$$

Putting everything together, the magnetic field dependence of $\mu_{2,0}$ is given by two Lorentzian resonances with half-widths $y_{1}$ and $y_{2}$ that are superposed on a (field independent) background:

$$
\mu_{2,0}=P_{0}+\frac{A_{1}}{\beta^{2}+y_{1}^{2}}+\frac{A_{2}}{\beta^{2}+y_{2}^{2}} .
$$

Although the quantities $P_{0}, A_{1}, A_{2}, y_{1}$, and $y_{2}$ can be written explicitly in terms of $\gamma_{1}, \gamma_{2}$, and $\theta$, the expressions are cumbersome and not very informative.

$$
\text { 2. } \Gamma_{0}^{(2)} \approx \Gamma_{1}^{(2)} \approx \Gamma_{2}^{(2)}
$$

To derive simple expressions one needs more information about the physical values of the parameters. Simpler expressions can be found when $\gamma_{1}=\Gamma_{1}^{(2)} / \Gamma_{0}^{(2)} \approx \gamma_{2}=\Gamma_{2}^{(2)} / \Gamma_{0}^{(2)} \approx$ 1 , a situation that can be modeled by parametrizing the 
relaxation rates as

$$
\begin{aligned}
& \gamma_{1}=1+s / 2+d / 2, \\
& \gamma_{2}=1+s / 2-d / 2,
\end{aligned}
$$

so that $\gamma_{1}-\gamma_{2}=d \ll 1$ and $\gamma_{1}+\gamma_{2}-2=s \ll 1$. For simplicity let us assume that the angle $\theta$ is at the magic value $\arccos (1 / \sqrt{3})$. In that case one finds

$$
\begin{aligned}
& P_{0}=0 \\
& y_{1}^{2}=\frac{1}{4}+\frac{5}{24} s+\frac{1}{72} d+\cdots,
\end{aligned}
$$

$$
\begin{aligned}
& y_{2}^{2}=1+\frac{2}{3} s-\frac{2}{9} d+\cdots, \\
& A_{1}=\frac{1}{12}+\frac{25}{216} s-\frac{55}{648} d+\cdots, \\
& A_{2}=\frac{2}{3}+\frac{7}{27} s+\frac{17}{81} d+\cdots
\end{aligned}
$$

For $s=d=0$, these expressions correspond to the result which can be inferred from Eq. (39) using the magic value of $\theta$.
[1] T. L. Gustavson, P. Bouyer, and M. A. Kasevich, Phys. Rev. Lett. 78, 2046 (1997).

[2] D. Budker and M. Romalis, Nat. Phys. 3, 227 (2007).

[3] J. Vanier and C. Audoin, The Quantum Physics of Atomic Frequency Standards (Hilger, Bristol and Philadelphia, 1989).

[4] W. Happer, Rev. Mod. Phys. 44, 169 (1972).

[5] K. Blum, Density Matrix Theory and Applications (Plenum, New York, 1996).

[6] U. Fano, Rev. Mod. Phys. 29, 74 (1957).

[7] A. Omont, Prog. Quantum Electron. 5, 69 (1979).

[8] S. M. Rochester and D. Budker, Am. J. Phys. 69, 450 (2001).

[9] http://demonstrations.wolfram.com/OpticalPumpingPopulation Dynamics/.
[10] http://demonstrations.wolfram.com/OpticalPumping VisualizationOfSteadyStatePopulationsAndPolari/.

[11] D. Budker, W. Gawlik, D. F. Kimball, S. M. Rochester, V. V. Yashchuk, and A. Weis, Rev. Mod. Phys. 74, 1153 (2002).

[12] H. S. Wall, Analytic Theory of Continued Fractions (Van Nostrand, Princeton, NJ, 1948).

[13] N. Castagna and A. Weis, Phys. Rev. A 84, 053421 (2011); 85, 059907(E) (2012).

[14] W. Happer, Phys. Rev. B 1, 2203 (1970).

[15] E. Breschi and A. Weis, Phys. Rev. A 86, 053427 (2012).

[16] J. J. Sakurai and Jim J. Napolitano, Modern Quantum Mechanics, 2nd ed. (Addison-Wesley, Reading, MA, 2010). 